\title{
Analysis of laser-induced heating in optical neuronal guidance
}

\author{
Ebbesen, Christian L.; Bruus, Henrik
}

Published in:

Journal of Neuroscience Methods

Link to article, DOI:

10.1016/j.jneumeth.2012.02.006

Publication date:

2012

Document Version

Publisher's PDF, also known as Version of record

Link back to DTU Orbit

Citation (APA):

Ebbesen, C. L., \& Bruus, H. (2012). Analysis of laser-induced heating in optical neuronal guidance. Journal of Neuroscience Methods, 209(1), 168-177. https://doi.org/10.1016/j.jneumeth.2012.02.006

\section{General rights}

Copyright and moral rights for the publications made accessible in the public portal are retained by the authors and/or other copyright owners and it is a condition of accessing publications that users recognise and abide by the legal requirements associated with these rights.

- Users may download and print one copy of any publication from the public portal for the purpose of private study or research.

- You may not further distribute the material or use it for any profit-making activity or commercial gain

- You may freely distribute the URL identifying the publication in the public portal

If you believe that this document breaches copyright please contact us providing details, and we will remove access to the work immediately and investigate your claim. 
Basic Neuroscience

\title{
Analysis of laser-induced heating in optical neuronal guidance
}

\author{
Christian L. Ebbesen ${ }^{\mathrm{a}, *}$, Henrik Bruus ${ }^{\mathrm{b}}$ \\ a Niels Bohr Institute, University of Copenhagen, Blegdamsvej 17, DK-2100 Copenhagen Ø, Denmark \\ ${ }^{\mathrm{b}}$ Department of Micro- and Nanotechnology, Technical University of Denmark, DTU Nanotech Bldg. 345 B, DK-2800 Kongens Lyngby, Denmark
}

\section{A R T I C L E I N F O}

\section{Article history:}

Received 4 April 2011

Received in revised form 2 February 2012

Accepted 4 February 2012

\section{Keywords:}

Optical neuronal guidance

Neuronal growth

Laser heating

Temperature gradient

Microfluidics

Biochip

\begin{abstract}
A B S T R A C T
Recently, it has been shown that it is possible to control the growth direction of neuronal growth cones by stimulation with weak laser light; an effect dubbed optical neuronal guidance. The effect exists for a broad range of laser wavelengths, spot sizes, spot intensities, optical intensity profiles and beam modulations, but it is unknown which biophysical mechanisms govern it. Based on thermodynamic modeling and simulation using published experimental parameters as input, we argue that the guidance is linked to heating. Until now, temperature effects due to laser-induced heating of the guided neuron have been neglected in the optical neuronal guidance literature. The results of our finite-element-method simulations show the relevance of the temperature field in optical guidance experiments and are consistent with published experimental results and modeling in the field of optical traps. Furthermore, we propose two experiments designed to test this hypotheses experimentally. For one of these experiments, we have designed a microfluidic platform, to be made using standard microfabrication techniques, for incubation of neurons in temperature gradients on micrometer lengthscales.
\end{abstract}

(c) 2012 Elsevier B.V. All rights reserved.

\section{Introduction}

Controlling the growth direction of neurons is an objective of scientific interest in neuroscience, medicine and bioengineering with promise of application to, e.g. in vivo nerve regeneration and in vitro formation of neuronal circuits for study of neuronal networks (Ehrlicher et al., 2002; Zeck and Fromherz, 2001). It is known that the neuronal growth cone is the primary neuronal organelle of axonal growth and pathfinding, acting as an integrator of the cellular environment (Kater and Guthrie, 1990; Chen and Cheng, 2009). It has also been shown that it is possible to alter the direction of the axonal growth with various chemical guidance cues (Wang and Poo, 2005; Hong et al., 2000; Henley et al., 2004; Henley and Poo, 2004; Nishiyama et al., 2003).

A curious new addition to the field of neuronal guidance is optical neuronal guidance (Carnegie et al., 2008, 2009; Ehrlicher et al., 2002; Graves et al., 2009; Higuchi et al., 2005, 2007; Mohanty et al., 2005; Stevenson et al., 2006; Mathew et al., 2010; Koch et al., 2004) reviewed in Franze and Guck (2010) and Stevenson et al. (2010). In 1991 it was first observed that Swiss 3T3 cells (mammalian fibroblast-like cells) would extend pseudopodia towards near-infrared light sources (Albrecht-Buehler, 1991), and in 2002 it was shown that a near-infrared laser spot placed on and in front

\footnotetext{
* Corresponding author.

E-mail addresses: laut@fys.ku.dk (C.L. Ebbesen), henrik.bruus@nanotech.dtu.dk (H. Bruus).
}

of the leading edge of growing PC12 and NG108 neurons enhanced the neuronal growth into the laser spot and resulted in laser-guided turns of the growth cone and increased growth speed (Ehrlicher et al., 2002).

A better understanding of optical neuronal guidance would make it possible to optimize the guidance for, i.e. medical and research applications, however the biophysical mechanism underlying the phenomenon is not understood as stated in recent studies and reviews: "Notably, the underlying mechanisms responsible for the light-cell interactions described above, have not been fully understood or are not known" (Mathew et al., 2010), "The underlying mechanisms of all these physical guidance cues remain unclear" (Franze and Guck, 2010), and "These experiments are surprising, and the underlying mechanisms are not fully understood" (Stevenson et al., 2010). The experimental studies of optical neuronal guidance reported in the literature (briefly summarized below and in Table 1) have all been sought explained in terms of biophysical mechanisms only involving different forms of optical gradient forces (acting either directly on the actin polymerization mechanism or on the filopodia itself), while neglecting temperature effects from laser-induced heating.

All experiments demonstrating optical neuronal guidance in the literature have been done on PC12 (rat neuron precursor cell line) or NG108 (immortalized mouse neuroblastoma rat glioma hybrid cell line) neurons. The neurons are cultivated in DMEM (Dulbecco's modified Eagle's medium) with 10\% fetal bovine serum (FBS), 20 units/mL penicillin, 20 units/mL streptomycin in a humidified atmosphere $\left(37^{\circ} \mathrm{C}, 5 \% \mathrm{CO} 2\right)$ (Carnegie et al., 2008; Ehrlicher et al., 
Table 1

List of parameters for optical neuronal guidance from the literature: Laser power $P_{\text {laser }}$, spot size $\left(w_{s}\right.$, range of $w_{s}$ or $w_{s} \times$ length), laser wavelength $\lambda$, and beam shape (Gaussian Spot GS or Line Trap LT).

\begin{tabular}{clll}
\hline$P_{\text {laser }}[\mathrm{mW}]$ & Spot size $[\mu \mathrm{m}]$ & $\lambda[\mathrm{nm}]$ & Beam shape \\
\hline $20-120$ & $w_{s}=2-8$ & 800 & $\mathrm{GS}^{\mathrm{a}}$ \\
$20-200$ & Unknown & 800 & $\mathrm{GS}^{\mathrm{b}}$ \\
$120-200$ & $2.5-20$ & 1064 & $\mathrm{LT}^{\mathrm{c}}$ \\
$9-25$ & $2.5-20$ & 780 & $\mathrm{GS}^{\mathrm{d}}$ \\
$8-22$ & $2.5-20$ & 1064 & $\mathrm{GS}^{\mathrm{d}}$ \\
$30-70$ & $1 \times 45$ & 1064 & $\mathrm{LT}^{\mathrm{e}}$ \\
$40-100$ & Large & 1064 & $\mathrm{GS}^{\mathrm{f}}$ \\
40 & 0.5 & $1064 / 1070$ & $\mathrm{GS}^{\mathrm{g}}$ \\
50 & 1 & $1064 / 1070$ & $\mathrm{GS}^{\mathrm{g}}$ \\
100 & $1 \times 40$ & $1064 / 1070$ & $\mathrm{LT}^{\mathrm{g}}$ \\
\hline
\end{tabular}

a Ehrlicher et al. (2002).

b Koch et al. (2004).

c Mohanty et al. (2005)

d Stevenson et al. (2006)

e Carnegie et al. (2008).

f Graves et al. (2009).

g Carnegie et al. (2009).

2002). $12 \mathrm{~h}$ prior to experimentation, the neurons are plated at low seeding density on thin glass slides or thin $5 \mu \mathrm{g} / \mathrm{cm}^{2}$ laminin coated glass slides. $4 \mathrm{~h}$ prior to experimentation, the medium is changed to DMEM with $1 \%$ FBS and the slides are placed in a $37^{\circ} \mathrm{C}$ temperature controlled microscope stage. In the seminal study done on PC12 and NG108 cells by Ehrlicher et al. (2002), the guidance was done with an $800 \mathrm{~nm}$ and $1064 \mathrm{~nm}$ laser and a spot diameter of approximately $2-16 \mu \mathrm{m}$. The laser power was measured to be between 20 and $120 \mathrm{~mW}$. Variations in the laser spot size were done by optically de-focussing the beam (for variations $<4 \mu \mathrm{m}$ ) and by scanning the beam at a frequency of $\approx 0.1 \mathrm{~Hz}$. Approximately one-fourth to one-half of the laser spot was placed on the lamellipodium and the remainder of the laser spot was placed ahead of the leading edge of the growth cone (i.e. with the highest laser intensity on or just in front of the growth cone membrane). With a success rate of $85 \%$ the growth cone turned towards the laser spot compared to only $20 \%$ in the control with an imaginary laser spot.

Clearly, optical neuronal guidance has been demonstrated across a broad range of laser wavelengths, spot sizes, spot intensities, beam shapes and beam modulations. Moreover, for the low laser-power in the optical guidance experiments, the magnitude of optical forces is minute (Ehrlicher et al., 2002), and although it has been hypothesized that the very low optical gradient forces played a role in steering the neuronal growth cones with optical line traps (Mohanty et al., 2005), this has later been disproven (Carnegie et al., 2008). This makes an explanation of the underlying mechanism based on optical gradient forces unlikely.

Instead, we suggest that the effect is due to a biochemical signaling cascade initiated by laser-induced heating of the cell membrane, a mechanism which we show in this paper is consistent with the reported experimental parameters. Previous estimates of this effect in the optical neuronal guidance experiments suggested a negligible temperature increase (Albrecht-Buehler, 1991; Carnegie et al., 2008, 2009; Ehrlicher et al., 2002; Graves et al., 2009; Higuchi et al., 2005, 2007; Mohanty et al., 2005; Stevenson et al., 2006; Mathew et al., 2010; Koch et al., 2004), but our more detailed simulations show a temperature increase of the order $1{ }^{\circ} \mathrm{C} / 100 \mathrm{~mW}$ of laser power, which is in agreement with experimental results and modelling from the field of optical trapping (Schönle and Hell, 1998; Braun and Libchaber, 2002; Peterman et al., 2003; Ebert et al., 2007). Furthermore, we find a temperature gradient of the order $1{ }^{\circ} \mathrm{C} /$ typical neuronal growth cone-radius.

In support of an explanation based on biochemical signaling cascade initiated by laser-induced heating of the cell membrane, we
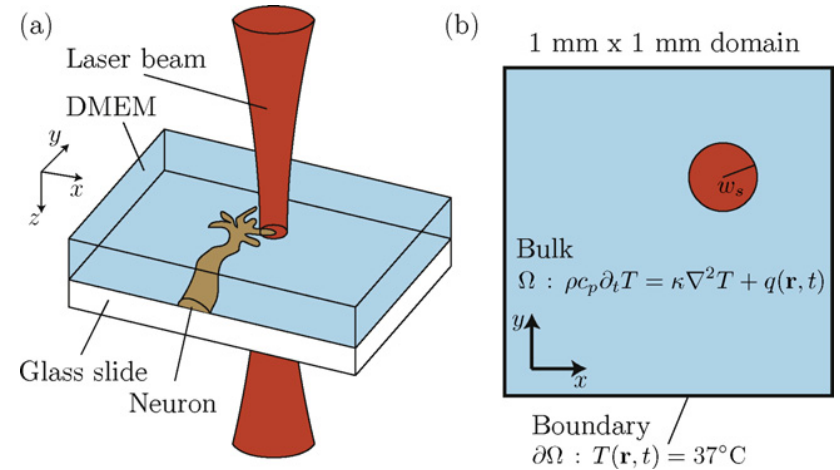

Fig. 1. (a) Sketch of optical guidance of a neuronal growth cone. (b) Simplified 2D model of a laser spot of spot size $w_{s} \approx 2 \mu \mathrm{m}$ in a horizontal $x y$-plane with fixedtemperature edges. The heat equation is also shown, including the laser heating term $q(\mathbf{r}, t)$.

turn to the following studies in the literature. It has been shown that chemical guidance of growth cones is mediated by an asymmetric influx of calcium ions $\mathrm{Ca}^{2+}$ (Henley and Poo, 2004) and the neuronal transient receptor potential (TRP) channels appear to be the key sensors and mediators in the process (Wang and Poo, 2005; Talavera et al., 2008; Li et al., 2005; Sappington et al., 2009). It is know that TRP channels can be activated by depolarization of the cell using chemical guidance factors or by an increase in temperature (Talavera et al., 2008; Caterina et al., 1997) and highly localized heating (Huang et al., 2010), and there is strong evidence in general that depolarization of the neuronal membrane is highly sensitive to small variations in thermodynamic variables like temperature and pressure (Wunderlich et al., 2009; Blicher et al., 2009; Andersen et al., 2009; Wodzinska et al., 2009). We therefore propose the hypothesis that the optical neuronal guidance is mediated by laser-induced heating, in particular by influx of $\mathrm{Ca}^{2+}$-ions due to heat-induced activation of neuronal TRP channels, discussed more thoroughly in Section 4.1.

The structure of our paper is the following. In Section 2 we present our thermal model and theory, and using it in Section 3 we predict by numerical simulation temperature increases above $1{ }^{\circ} \mathrm{C}$ due to heating by absorption of infra-red laser light. In Section 4 we discuss the implication for heat-dependent biochemical processes important for neuronal growth, and finally in Section 5 we propose designs of experiments to be carried out to test our hypothesis.

\section{Thermal model and theory}

\subsection{Governing equations}

To calculate the laser-induced heating of a sample during a typical optical neuronal guidance experiment, see Fig. 1(a), we use Fourier's heat equation,

$\rho c_{p} \partial_{t} T=\kappa \nabla^{2} T+q(\mathbf{r}, t)$

where $\rho$ is density, $c_{p}$ constant-pressure heat capacity, $\kappa$ thermal conductivity, and $q(\mathbf{r}, t)$ is a source term describing the heating (dissipated energy per volume per time) due to the incident laser light. Parameter values are listed in Table 2.

The optical intensity $I_{0}$ of a Gaussian laser beam in free space is given by

$I_{0}(\mathbf{r}, t)=\frac{2 P_{\text {laser }}}{\pi w^{2}(z)} \exp \left[-\frac{2 a^{2}(\mathbf{r}, t)}{w^{2}(z)}\right]$, 
Table 2

Material parameters for water and pyrex glass: density $\rho$, heat capacity $c_{p}$, heat conductivity $\kappa$, and absorption coefficient $\alpha$ (Lide, 2003; Bruus, 2008). The laser wavelengths are listed for specific values of $\alpha$ in water.

\begin{tabular}{lclll}
\hline & $\rho\left[\mathrm{kg} / \mathrm{m}^{3}\right]$ & $c_{p}[\mathrm{~J} / \mathrm{kg} \mathrm{K}]$ & $\kappa[\mathrm{W} / \mathrm{Km}]$ & $\alpha\left[\mathrm{m}^{-1}\right]$ \\
\hline Water & 998 & 4180 & 0.62 & \\
$540 \mathrm{~nm}$ & & & & 0.047 \\
$780 \mathrm{~nm}$ & & & 2.7 \\
$800 \mathrm{~nm}$ & & & 2.2 \\
$1064 \mathrm{~nm}$ & & & & 15 \\
$1200 \mathrm{~nm}$ & & 840 & 1.0 & 127 \\
Pyrex glass & 2230 & & & $<0.01$ \\
\hline
\end{tabular}

where $P_{\text {laser }}$ is the total laser power and $a(\mathbf{r}, t)$ is the radial distance from the beam center, which is only time dependent, if the laser is scanning over the sample. The beam width $w(z)$ is given by,

$w(z)=w_{0} \sqrt{1+\left(\frac{\lambda z}{\pi w_{0}^{2}}\right)^{2}}$,

where $\lambda$ is the wavelength and $w_{0}$ is the beam waist at the laser objective. Due to absorption in the medium, the intensity $I$ will decay exponentially in the $z$-direction,

$\partial_{z} I(\mathbf{r}, t)=-\alpha I(\mathbf{r}, t)$

where $\alpha$ is the absorption coefficient. In the liquid domain, we neglect possible higher absorbance of both medium and the neuron itself relative to that of pure water rendering the calculation a lower bound to actual temperature increase. In the literature, the most used wavelengths are $800 \mathrm{~nm}$ and $1064 \mathrm{~nm}$. Table 2 lists absorption coefficients for water and pyrex glass at these wavelengths. Due to the temperature controlled stage, we will simply require that the bottom of the glass slide and the sides of the cell culture dish is kept at $37^{\circ} \mathrm{C}$,

$T(t)=37^{\circ} \mathrm{C}$, (heated stage boundary).

For the water/air interface at the top, we will allow heat exchange with the surrounding air, modeled by the boundary condition,

$\kappa \mathbf{n} \cdot \nabla T=h\left(T-T_{\text {air }}\right)$, (air boundary),

where $T_{\text {air }}$ is the temperature of the surrounding air and the material parameter $h=7.5 \mathrm{~W} /\left(\mathrm{m}^{2} \mathrm{~K}\right)$.

\subsection{Simplified models}

\subsubsection{Stationary laser spot in $3 D$}

The heat equation was solved numerically using a finite element method. There are several relevant length scales in this problem: The geometry of the glass slide with the $37^{\circ} \mathrm{C}$ boundary has centimeter dimensions and the axon and spot size are on the order of micrometer, a length-scale mismatch making a full 3D simulation computer-memory demanding. For a stationary laser spot, the rotational symmetry of the spot makes the temperature field axisymmetric and the problem reduces to a $2 \mathrm{D}$ problem in the radial coordinate $R$ and the vertical coordinate $z$. This enables numerical simulations on the computers at our disposal. In contrast, the motion of a scanning laser spot breaks the axisymmetry of the system, and the resulting full 3D problem is beyond the capacity of our computer hardware, forcing us to restrict the analysis to the horizontal $x y$-plane, see Section 2.2.2.

For a stationary laser spot, we model the system as $1.5 \mathrm{~mm}$ thick and $3 \mathrm{~mm}$-radius cylindrical water domain on a $0.3 \mathrm{~mm}$ pyrex glass slide, see Section 3.1. The center of the laser spot is placed on the cylinder axis. The slide is placed in a heated microscope stage with the bottom and sides kept at a fixed temperature of $37^{\circ} \mathrm{C}$. Having the water/glass interface at $z=0$, the boundary conditions become,

$T(R, z)=37^{\circ} \mathrm{C}$, for $z=-0.3 \mathrm{~mm}$, (bottom of glass slide)

$T(R, z)=37^{\circ} \mathrm{C}$, for $R=3 \mathrm{~mm}$. (sides of water domain)

The boundary condition at the water/air interface at $z=3 \mathrm{~mm}$ is given the water/air heat exchange expression given in Eq. (6) with $T_{\text {air }}=37^{\circ} \mathrm{C}$.

\subsubsection{Scanning laser spot in the $x y$-plane}

For a scanning laser spot, we used that the total height of the glass slide and medium is only a few millimeter, while the absorption coefficients in water are on the order of centimeter. From our 3D simulation, we notice that there is almost no laser power dissipation in the pyrex glass slide, and that the constant-temperature boundary condition at the bottom of the glass slide only affects the temperature field very close to the glass/water interface. This means, as discussed in Section 3.1, that we can reduce the model to a laser spot moving in a horizontal 2D water domain, see Fig. 1(b). The dissipated power per volume is

$\frac{P}{\mathcal{V}}=\left|\partial_{z} I(\mathbf{r}, t)\right|=q(\mathbf{r}, t)$.

Because the absorption-length of laser light is on the order of centimeter, the $z$-dependence of the source term is negligible in the bulk water domain $\Omega$,

$$
\begin{aligned}
q(\mathbf{r}, t) & =\frac{2 \alpha P_{\text {laser }}}{\pi w_{s}^{2}} \exp \left[-\frac{2 a^{2}(\mathbf{r}, t)}{w_{s}^{2}}-\alpha z\right] \\
& \approx \frac{2 \alpha P_{\text {laser }}}{\pi w_{s}^{2}} \exp \left[-\frac{2 a^{2}(\mathbf{r}, t)}{w_{s}^{2}}\right], \text { for } \mathbf{r} \text { in } \Omega,
\end{aligned}
$$

where $w_{s}$ is the radius of the laser spot in the 2D plane of the glass slide and $a(\mathbf{r}, t)$ is the distance from the center of the laser spot. In the $2 \mathrm{D}$ geometry, we only have $\mathbf{r} \equiv(x, y)$. If the laser spot center coordinates move in time with a trajectory $\mathbf{r}_{c}(t)=\left(x_{c}(t), y_{c}(t)\right)$, we have

$a^{2}(\mathbf{r}, t)=\left(x-x_{c}(t)\right)^{2}+\left(y-y_{c}(t)\right)^{2}$.

Furthermore, we will also make the simplification of only calculating the temperature field in a $1 \mathrm{~mm} \times 1 \mathrm{~mm}$ square water domain, as we found this domain large enough to resolve the temperature field around the laser without seeing any edge-effects from the boundary. In the simplified 2D model, the boundary conditions simplify to the one requirement that the temperature $T$, at the boundary $\partial \Omega$, is kept constant at the temperature of the temperature controlled microscope stage,

$T(\mathbf{r}, t)=37^{\circ} \mathrm{C}$, for $\mathbf{r}$ at $\partial \Omega$.

\section{Results}

To simulate the effects of heating in an optical guidance experiment, the temperature field was calculated using the COMSOL finite element method software (COMSOL, 2011). The heat equation with the above-mentioned boundary conditions was solved for a stationary laser spot in the full 3D model as well as for stationary and scanning laser spot in the reduced 2D model.

\subsection{D temperature field for a stationary laser spot}

The 3D steady-state temperature field was calculated for a stationary 1064-nm laser spot with $w_{s}=1 \mu \mathrm{m}$ and $P=100 \mathrm{~mW}$, see Fig. 2(a)-(c). After a mesh convergence analysis, the domain was finally meshed with a maximum mesh element size of $1 \mu \mathrm{m}$ for $R<30 \mu \mathrm{m}$ and $20 \mu \mathrm{m}$ for $R>30 \mu \mathrm{m}$. Because a typical height of a 
(a)

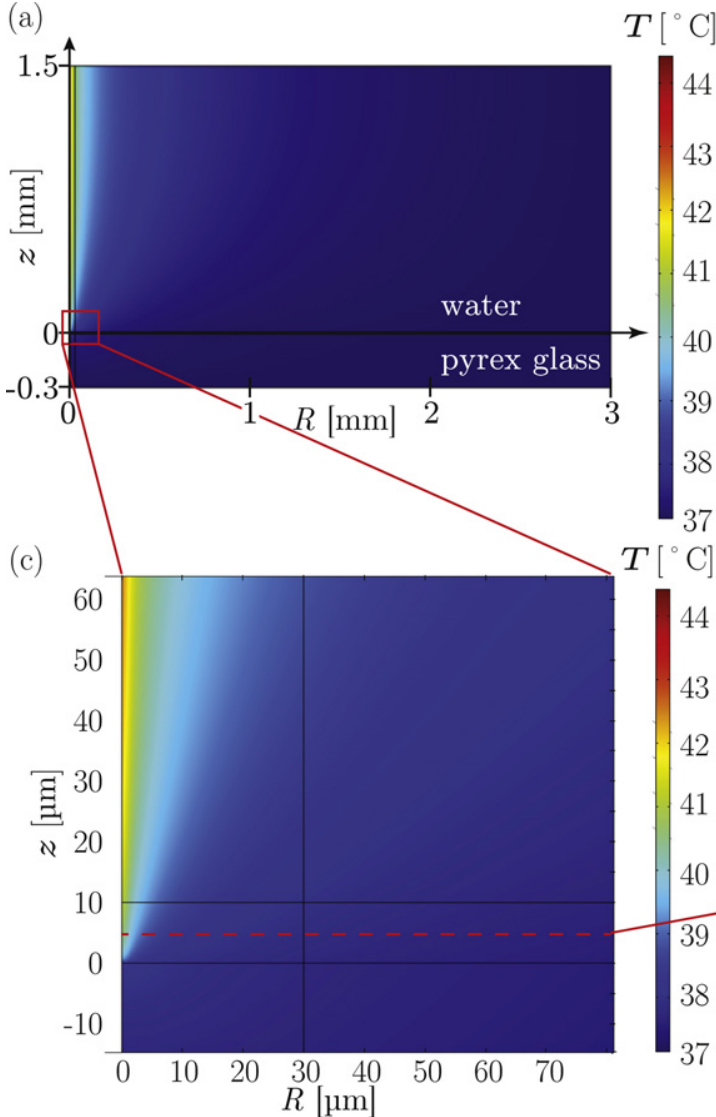

(b)

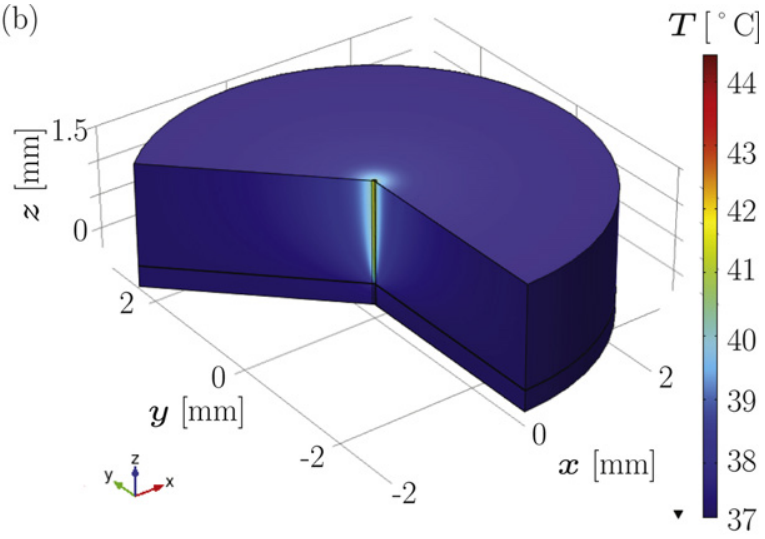

(d)

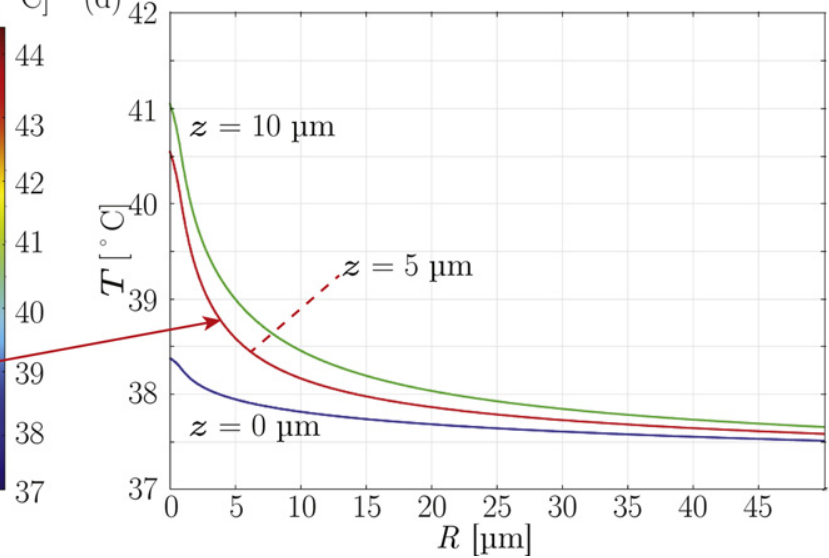

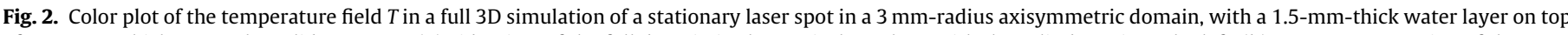

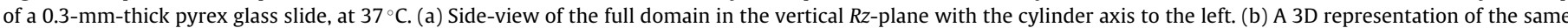

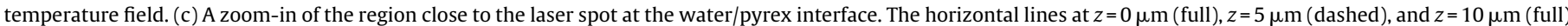

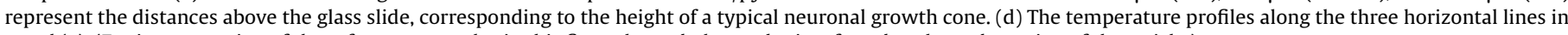
panel (c). (For interpretation of the references to color in this figure legend, the reader is referred to the web version of the article.)

neuronal growth cone is $10 \mu \mathrm{m}$ (Ehrlicher et al., 2002), the radial temperature profiles at $z=0,5,10 \mu \mathrm{m}$ above the glass slide were plotted, see Fig. 2(d), and it was found, that the gradient of the temperature field was approximately $0.6,2.3$ and $2.6^{\circ} \mathrm{C} / 10 \mu \mathrm{m}$, respectively. This shows, that even when the bottom of the glass slide is kept fixed at $37^{\circ} \mathrm{C}$ in a thermocontrolled chamber, irradiation by a near-infrared laser will still give rise to a biologically significant temperature difference of about $1{ }^{\circ} \mathrm{C}$ across the width one neuronal growth cone, approximately one growth-cone-height above the glass slide. These results further imply that presence of the glass slide with a fixed-temperature bottom has a negligible influence on the temperature field already a few micrometer above the water/pyrex interface. This allows us to reduce the numerical simulations to a horizontal 2D-plane in water with the edges kept at $37^{\circ} \mathrm{C}$, see Fig. 1 (b) and the following subsection.

\section{2. $2 D$ temperature field for a stationary laser spot}

From now on we analyze a horizontal 2D water domain with a fixed-temperature boundary. The reduction in dimensionality allows us to break the symmetry and study a stationary or scanning laser spot at arbitrary positions in square domain, see Fig. 1(b).

Initially, a stationary laser spot was modeled. To show that the symmetry of the boundaries does not affect the symmetry of the temperature profile around the laser spot, the latter was placed slightly off center in the simulations, see Fig. 3(a). To characterize the temperature field, we define three values: the temperature $T_{S}$ in the center of the laser spot, the temperature $T_{30}$ at a distance $30 \mu \mathrm{m}$ away from the center of the laser spot, and the distance $d_{\mathrm{hm}}$ from the maximum temperature to the temperature at half-maximum approximating the half-width of the temperature profile, Fig. 3(b). We choose $30 \mu \mathrm{m}$, as this represents a typical neuronal growth cone width (Ehrlicher et al., 2002), so if the laser spot is kept at one leading edge of the growth cone, $T_{S}-T_{30}$ will give an estimate of the temperature difference across the growth cone. In Fig. 4, we show $T_{S}$ and $T_{30}$ for a spot with $w_{s}=2 \mu \mathrm{m}$ at two typically used wavelengths. As expected, we see that the temperature field depends linearly on the laser power. We also see a clear temperature gradient across the growth cone. For $P=100 \mathrm{~mW}$ at the two calculated wavelengths, we find the temperature rise

$$
\begin{array}{cc}
1064 \mathrm{~nm}: & T_{S}-T_{0}=2.18^{\circ} \mathrm{C}, \\
& T_{30}-T_{0}=1.15^{\circ} \mathrm{C}, \\
800 \mathrm{~nm}: & T_{S}-T_{0}=0.32^{\circ} \mathrm{C}, \\
& T_{30}-T_{0}=0.17^{\circ} \mathrm{C} .
\end{array}
$$

This magnitude of heating is in agreement with experimentally observed laser-induced heating of vesicles by infrared optical tweezers (Liu et al., 1995), of DNA in an infrared optical trap (Braun and Libchaber, 2002), of micron-sized silica and polystyrene beads in a optical traps (Peterman et al., 2003), and temperature sensitive dyes in buffer suspension in an infrared dual-beam laser trap (Ebert et al., 2007). We find that for spot sizes with $w_{s}=1-2 \mu \mathrm{m}$, we have $d_{\mathrm{hm}}=32.7 \mu \mathrm{m}$, which is comparable to the diameter of a neuronal growth cone. 


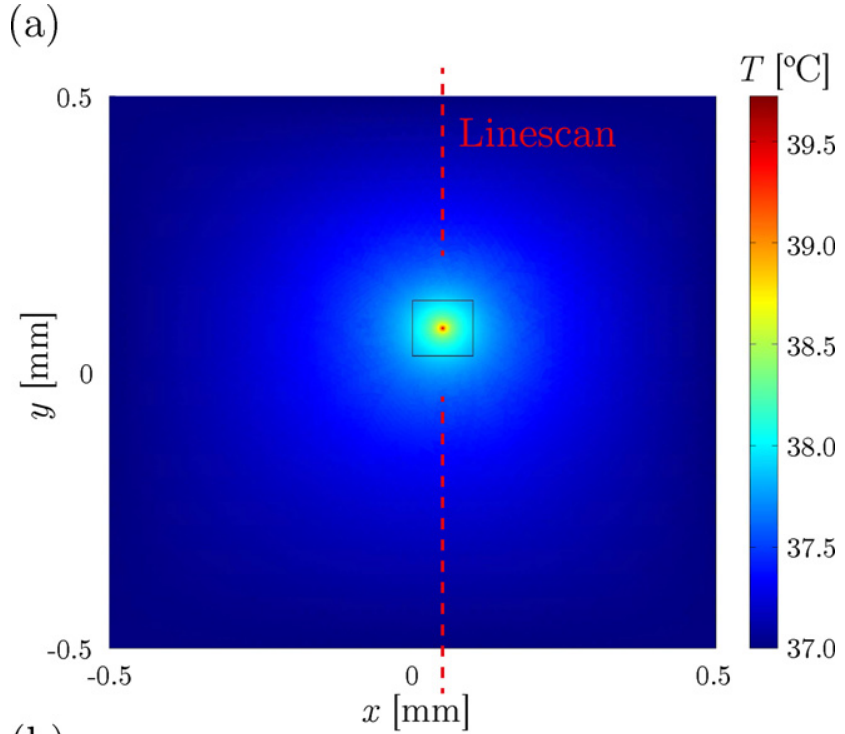

(b)

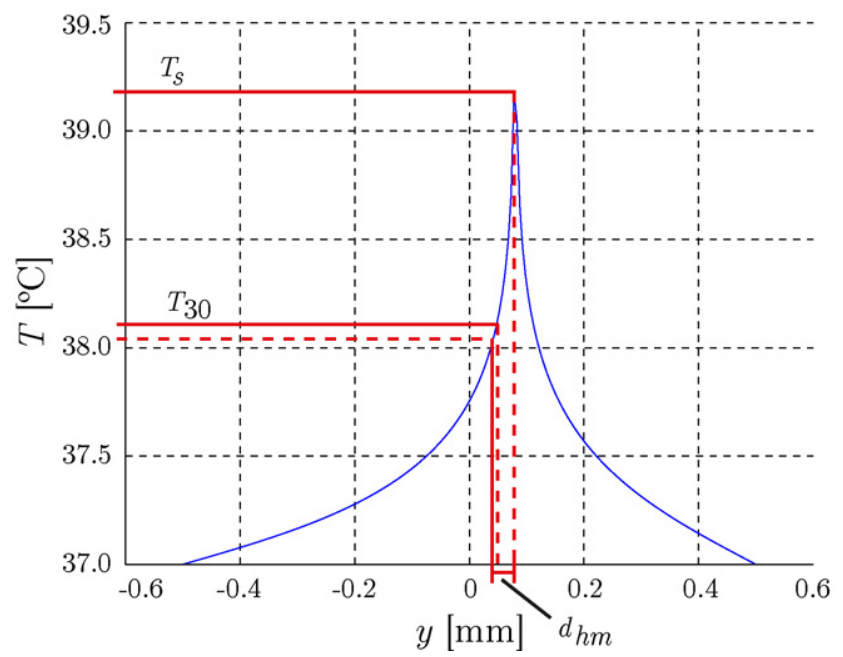

Fig. 3. (a) Steady-state temperature field $T(\mathbf{r})$ from an off-centered, stationary, 1064nm-wavelenght laser spot with $w_{s}=2 \mu \mathrm{m}$ and $P=100 \mathrm{~mW}$. (b) A line scan of $T$ along the red dotted line of panel (a). Definitions are given of spot temperature $T_{s}$, temperature $T_{s}$ at $30 \mu \mathrm{m}$, and distance $d_{\mathrm{hm}}$ from max to half-max temperature. The mesh element size around the laser spot center is $1 \mu \mathrm{m}$, which is sufficient to resolve the temperature profile of the Gaussian spot, see also the inset of Fig. 6. (For interpretation of the references to color in this figure legend, the reader is referred to the web version of the article.)

\section{3. $2 D$ temperature field for a scanning laser spot}

In some experiments, the neurons were stimulated with a scanning laser to achieve a larger functional laser spot (Ehrlicher et al., 2002). The largest spots reported in the literature have a diameter of $16 \mu \mathrm{m}$, so we calculated the temperature field from harmonically scanning a $2-\mu \mathrm{m}$-diameter laser spot along a $16-\mu \mathrm{m}$ path with a frequency of $0.1 \mathrm{~Hz}$. In Fig. 5 is shown the temperature field after $120 \mathrm{~s}$ with a scanning and non-scanning laser along the scan axis. As initial condition at $t=0$ was used that the temperature everywhere was $T_{0}(\mathbf{r})=T(\mathbf{r}, 0)=37^{\circ} \mathrm{C}$. We see a $20 \%$ reduction in the temperature field along the scanning axis, but there are still definite temperature gradients over distances comparable to neuronal growth cone sizes. We also observe that the temperature field follows the laser position in time (data not shown). Scanning the laser with $0.1 \mathrm{~Hz}$ does not make one large uniformly heated spot; the steep temperature gradients around the scanning laser are still present. This can

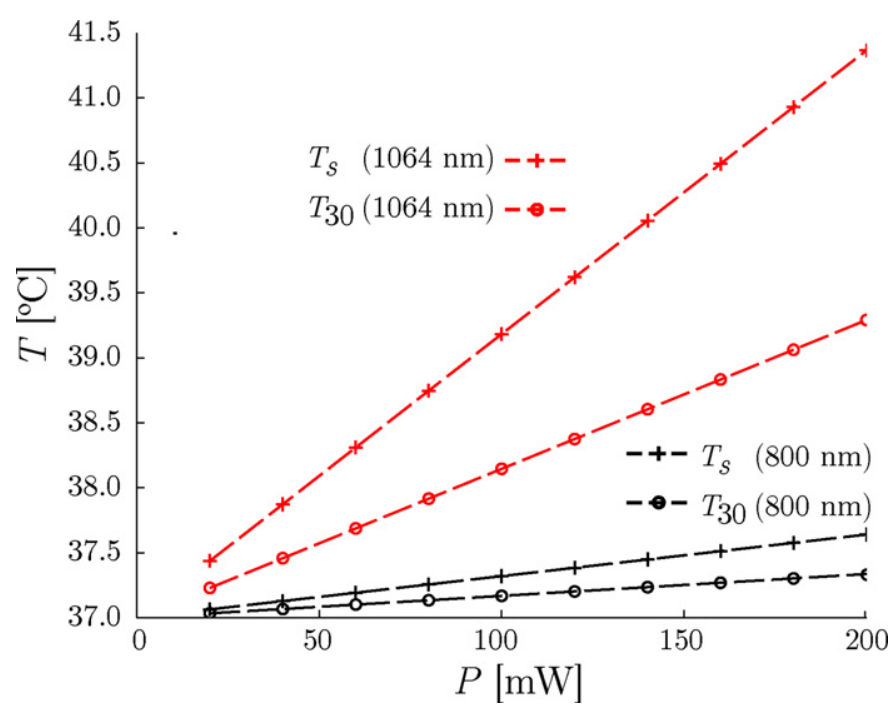

Fig. 4. Spot temperature $T_{s}$ and temperature $T_{30}$ at $30 \mu \mathrm{m}$, versus incident laser power $P$ for a laser spot with $w_{s}=2 \mu \mathrm{m}$ at wavelengths $800 \mathrm{~nm}$ (black) and $1064 \mathrm{~nm}$ (red). (For interpretation of the references to color in this figure legend, the reader is referred to the web version of the article.)

be explained by thermal diffusion in the water domain with the diffusion constant $D_{\text {th }}$,

$D_{\text {th }}=\frac{\kappa}{\rho c_{p}}=1.49 \times 10^{-7} \mathrm{~m}^{2} \mathrm{~s}^{-1}$.

Scanning at $f=0.1 \mathrm{~Hz}$ gives a characteristic diffusion length $\ell_{\text {th }}$,

$\ell_{\mathrm{th}}=\sqrt{D_{\mathrm{th}} / f}=1.22 \mathrm{~mm} \gg w_{s}$.

From this it is clear that the temperature field will follow the laser spot and quickly diffuse away from any regions with no incident laser power. We can also calculate the time it will take the laser spot to heat up. We can approximate,

$\rho c_{p} \partial_{t} T=\kappa \nabla^{2} T \approx \kappa \frac{T-T_{0}}{\ell_{T}^{2}}$,

where $T_{0}$ is the temperature of the bulk, and $\ell_{T}$ is a characteristic length on which $T$ changes from $T_{0}$. Setting $\ell_{T}=2 d_{\mathrm{hm}}$ gives a time constant,

$\tau=\ell_{T}^{2} / D_{\text {th }}=27.6 \mathrm{~ms}$.

This also shows that the temperature field will indeed follow the relatively slowly moving laser spot.

\subsection{Validation of the numerics}

To ensure that the finite-element-method mesh was sufficiently fine to resolve the geometry, we investigated the convergence of the numerical solution in several ways. To resolve the temperature field close to the laser spot, the mesh in a $100 \mu \mathrm{m} \times 100 \mu \mathrm{m}$ domain around the laser spot was much finer than the mesh in the rest of the bulk domain. We found it sufficient to use maximum element sizes of $1 \mu \mathrm{m}$ and $25 \mu \mathrm{m}$, respectively. We calculated $T_{S}$ for an increasingly fine mesh to make certain that the solution was stable. A mesh analysis showed convergence for maximum element size below $2 \mu \mathrm{m}$ (data not shown). This result makes sense as the Gaussian spot has a width of $\sim 5 \mu \mathrm{m}$.

Another validation of the numerics is a comparison with an approximate analytical solution. Outside the laser spot $q(\mathbf{r}, t)=0$, so the steady-state version of equation Eq. (1) $\left(\partial_{t} T=0\right)$ reduces to Laplace's equation in $2 \mathrm{D}, \nabla^{2} T=0$ with axial symmetry around the 

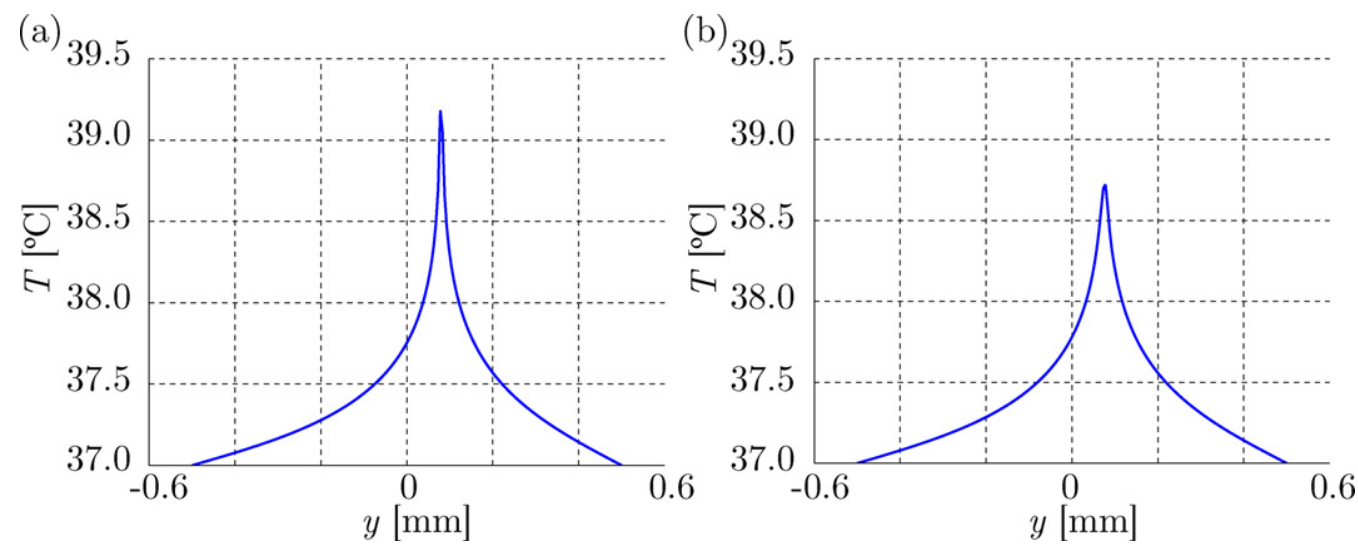

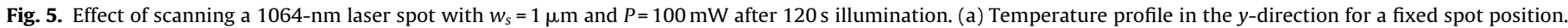
(b) Similar, but now oscillating the spot position harmonically by $\pm 7 \mu \mathrm{m}$ in the $y$-direction (along the scanning path) with a frequency of $0.1 \mathrm{~Hz}$, see Fig. 3 .

laser spot. It is easily checked that an analytical solution in this case is

$T(\mathbf{r})=A \ln \left|\mathbf{r}-\mathbf{r}_{C}\right|+\mathbf{B} \cdot\left(\mathbf{r}-\mathbf{r}_{C}\right)+C$,

where $\mathbf{r}_{C}$ is the position of the center of the laser spot, $A$ and $C$ are constants, and $\mathbf{B}$ is a constant vector. This corresponds to the temperature field around a point heat source. Plotting Eq. (19) alongside the numerical solution we find very good agreement, which is another indication that the finite element method is sufficiently meshed, see Fig. 6.

\section{Discussion}

For both stationary and scanning micrometer-sized, $100 \mathrm{~mW}$ infrared laser spots, we have found that biologically significant temperature differences (about $1^{\circ} \mathrm{C}$ ) on the length scale (about $10 \mu \mathrm{m}$ ) of a neuronal growth cone arise, even when the experiment is conducted in a setup with bottom and side-walls kept at $37^{\circ} \mathrm{C}$. Our simulations represent a lower limit of the heating effects, as we have neglected possible higher absorbance of both medium and the neuron itself relative to that of pure water. Moreover, we have

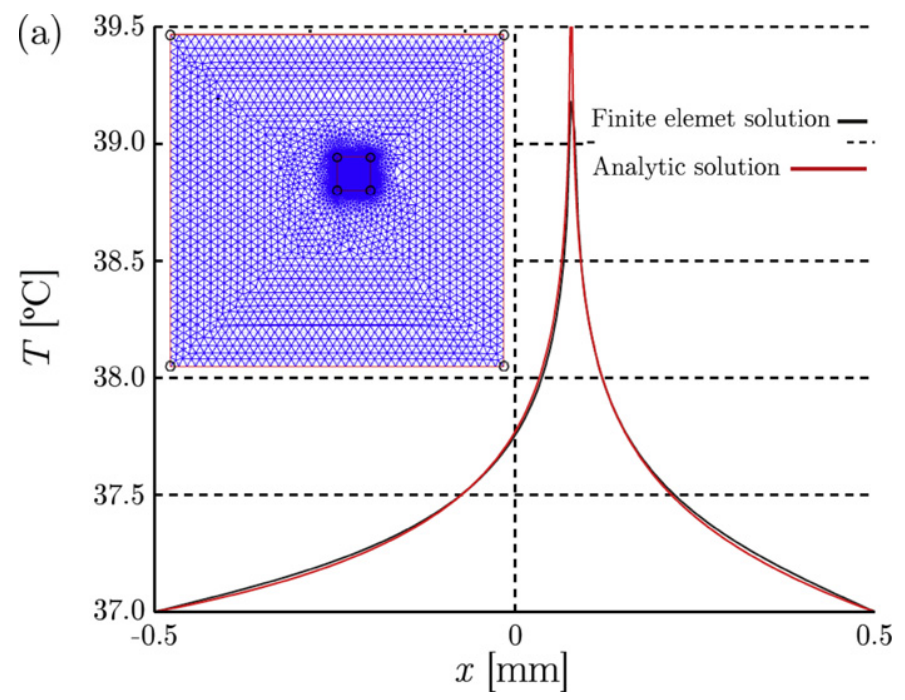

Fig. 6. (a) Comparison between shape of the finite-element-method solution (black) and the analytical expression Eq. (19) (red). The solution was computed for a 1064nm laser spot with $w_{s}=1 \mu \mathrm{m}$ and $P=100 \mathrm{~mW}$ and the inset shows the mesh with a high resolution near the laser spot. A maximum element size of $0.5 \mu \mathrm{m}$ corresponds to $2.3 \times 10^{5}$ degrees of freedom. (For interpretation of the references to color in this figure legend, the reader is referred to the web version of the article.) assumed ideal fixed temperature at the bottom of the glass slide in the microscope stage, where in real experiments the access hole for the optics reduces the thermal conductivity of the stage, and subsequent increased laser heating effects.

We therefore propose in the following that the optical neuronal guidance is mediated by laser heating activation of temperature sensitive TRP channels and discuss previously proposed hypotheses involving optical gradient forces and temperature effects in actin polarization. We also show, that the heat-activation hypotheses is consistent with other examples of optical cellular guidance.

\subsection{Potential TRP channel activation in optical neuronal guidance}

It is known that the neuronal growth cone uses surface receptors to integrate the cellular environment and mediate the growth of the axon. It has been shown that cytoplasmic $\mathrm{Ca}^{2+}$ signals play a key part in this signaling, and that the concentration of intracellular $\mathrm{Ca}^{2+}$ is an important regulator of neurite growth (Wang and Poo, 2005; Hong et al., 2000; Henley et al., 2004; Henley and Poo, 2004; Nishiyama et al., 2003). Localized increases in $\mathrm{Ca}^{2+}$ concentration result in protrusion of filopodia and growth cone turning. Similarly, in the presence of $\mathrm{a} \mathrm{Ca}^{2+}$ ionophore, neurons will grow up along a $\mathrm{Ca}^{2+}$ gradient, presumably caused by a gradient of $\mathrm{Ca}^{2+}$ influx across the growth cone (Talavera et al., 2008).

The $\mathrm{Ca}^{2+}$ regulated motility has been observed to have a bimodal nature, where a gradient of $\mathrm{Ca}^{2+}$ influx across the growth cone will mediate steering responses, with higher amplitude $\mathrm{Ca}^{2+}$ signals (steep gradients) mediating attraction and lower amplitude $\mathrm{Ca}^{2+}$ signals (gentle/gradual gradients) mediate repulsion. A moderate increase in the overall cytoplasmic $\mathrm{Ca}^{2+}$ concentration can initiate or promote axonal motility and extension (Henley and Poo, 2004).

The TRPV subfamily, dubbed thermoTRPs, is highly sensitive to temperature, and essentially serve as cellular membrane "thermometers" (Talavera et al., 2008). Strong heat is detected by activation of TRPV1 and TRPV2 channels (thresholds of $\approx 42{ }^{\circ} \mathrm{C}$ and $\approx 50^{\circ} \mathrm{C}$, respectively) and warmth (minor temperature increases compared to body temperature) is sensed by TRPV3 and TRPV4 channels. These two channels exhibit significant activity at body temperature, and are further activated by increases in temperature in the warm range (Caterina, 2007; Talavera et al., 2008). That is, hippocampal neurons are known to express TRPV4, allowing influx of cations at $37^{\circ} \mathrm{C}$ increasing excitability of these neurons (Talavera et al., 2008) and TRPV1 can carry significant currents at $37^{\circ} \mathrm{C}$, and thus potentially sense small fluctuations around body temperature (Szallasi et al., 2007).

It is not known, if the calculated temperature gradients are strong enough to cause steering of the neuronal growth cone. There 
are no reports on neuronal growth in temperature microgradients in the literature, but it is known that bulk neuronal development is temperature-dependent (Ishida and Cheng, 1991; Fukui et al., 1992), and that some organisms (nematodes) have been shown to be extremely temperature sensitive, exhibiting thermotaxis up temperature gradients as small as $10^{-4}{ }^{\circ} \mathrm{C} / \mathrm{cm}$ (Pline et al., 1988). It also seems that depolarization of the neuronal membrane is highly dependent on small variations in thermodynamic variables like temperature (Wunderlich et al., 2009; Blicher et al., 2009; Andersen et al., 2009; Wodzinska et al., 2009).

The optical guidance has been shown to work over a range of wavelengths $(760 \mathrm{~nm}, 800 \mathrm{~nm}, 1064 \mathrm{~nm}, 1070 \mathrm{~nm})$ and at different optical beam shapes. This is consistent with heating effects, which are essentially independent of specific wevelengths or beam shapes. Also, a curious effect is described in the literature: if the optical guidance is performed with a too high laser power, the cells retract instead of grow into the beam (Koch et al., 2004). This cannot be explained by a hypothesis only considering optical forces, but is consistent with the TRP activation hypothesis; localized heating induces $\mathrm{Ca}^{2+}$ influx and the $\mathrm{Ca}^{2+}$ signal mediated growth cone steering is known to be bimodal: moderate increases in cytoplasmic $\mathrm{Ca}^{2+}$ concentration can initiate or promote growth cone motility and extension, whereas high cytoplasmic $\mathrm{Ca}^{2+}$ concentration can inhibit growth and cause repulsion (Henley and Poo, 2004).

\subsection{Experiments with optical line traps}

One paper reports the use of optical line traps to steer the growth (Carnegie et al., 2008). It was investigated whether the optical gradient had an influence on the steering efficiency. The guidance was performed at $37^{\circ} \mathrm{C}$ on the NG108 cell line with a $30-70 \mathrm{~mW}$, $1064 \mathrm{~nm}$ laser with a spot size of approximately $1 \mu \mathrm{m} \times 45 \mu \mathrm{m}$. Three spot shapes were investigated: a line trap with a forward bias (e.g. forward optical intensity gradient), a line trap with a reverse bias, and a symmetric Gaussian shape. Similar levels of guidance are found regardless of the direction of the intensity variation along the line trap. Also, the same levels can be reached with a symmetric Gaussian beam shape. The line trap is prepared by blocking part of the laser beam path which reduces the total incident power on the sample (Carnegie et al., 2008). This means that the symmetric spot will deliver more power, while the biased spots will deliver less power, so a symmetric line is expected to increase the bulk growth speed more than the forward or backward biased lines. Also, the maximum optical intensity was always kept at the leading edge of the growth cone, so the reverse bias configuration should raise the temperature of the neuron more than the forward bias configuration. This is consistent with the measured average growth rates by Carnegie et al. (2008) using the three beam shapes: $65 \pm 11 \mu \mathrm{m} / \mathrm{h}$ (forward bias), $78 \pm 9 \mu \mathrm{m} / \mathrm{h}$ (reversed bias), and $111 \pm 11 \mu \mathrm{m} / \mathrm{h}$ (symmetric shape).

\subsection{Temperature effects in actin polarization}

It has also been proposed that the laser spot gradient forces are somehow "pooling" G-actin monomers in the cytoplasm to one side of the growth cone, thereby increasing the local actin concentration, thereby increasing the speed of actin polymerization on that side and thus steering the growth cone (Ehrlicher et al., 2002). The hypothesis seems to assume that in vivo actin polymerization is mainly a diffusion-driven process that depends strongly on the local concentration of G-actin monomers. However, it has been shown in experiments with in vitro $\mathrm{Mg}^{2+}$-induced polymerization of rabbit skeletal muscle G-actin that the actin polymerization is far too temperature dependent to be a strictly diffusion controlled process, i.e. does the rate constant $k_{+e}$ of elongation of actin filaments increase six-fold with a temperature increase from $10^{\circ} \mathrm{C}$ to $30^{\circ} \mathrm{C}$ (Zimmerle and Frieden, 1986).

It is also known that actin polymerization has a curious temperature dependence, where the fraction $\Phi$ of monomers polymerized has a constant value $\approx 0.1$ as a function of temperature until the temperature rises to $T_{p}$, where $\Phi$ rises to $\approx 0.8$ and then decreases again (Niranjan et al., 2001). This is derived from a system of four coupled differential equations (chemical equilibria), and $\Phi(T)$ is approximately a concave-down parabola of width $\approx 32 \mathrm{~K} . T_{p}$ is dependent on salt concentration, $[\mathrm{KCl}]$, and $\mathrm{G}$-actin concentration $\left[G_{0}\right]$. For growing neurons, we can assume approximate values of $\left[\mathrm{Cl}^{-}\right]=8.2 \pm 0.6 \mathrm{mM}$ (Irie et al., 1998) and $\left[\mathrm{G}_{0}\right]=16 \mu \mathrm{m}$ (Atkinson et al., 2004). Using that G-actin is a $42 \mathrm{kDa}$-protein, we find $T_{p} \approx 35^{\circ} \mathrm{C} . T_{p}\left(\left[\mathrm{G}_{0}\right]\right)$ is linear with a negative slope, so we have that for physiological temperatures and ion strengths, an increase in actin polymerization is equally likely to be due to an increase in temperature, as to an increase in $\mathrm{G}$-actin concentration. It even seems that for physiological conditions, $\Phi$ is much more sensitive to temperature variations, than to changes in G-actin concentration (Niranjan et al., 2001). However, we do not believe either optical gradient force mediated actin monomer pooling or the temperature dependence of actin polymerization to be able to account for the growth cone steering effects of optical neuronal guidance.

\subsection{Other examples of optical cellular guidance}

Besides its use in neuronal guidance, optical cellular guidance has been demonstrated on Swiss 3T3 cells in a seminal paper reporting extension of pseudopodia into infrared laser spots (Albrecht-Buehler, 1991). This is consistent with the hypothesis that the laser spot heating thermodynamically activates the TRP channels. It has been shown that cultured fibroblasts (NIH3T3), neuronal cells (NG108-15) and keratinocytes (M308) all express TRPV3 and TRPV4 and respond to warmth in a normal bath solution containing $\mathrm{Ca}^{2+}$ solution, but not in $\mathrm{Ca}^{2+}$-free solution, suggesting a primary role of TRPV regulated $\mathrm{Ca}^{2+}$ influx in cellular thermosensing (Mochizuki-Oda et al., 2007).

In the study on Swiss 3T3 cells, it was found that the cells would extend pseudopodia towards laser spots with $\lambda \geq 600 \mathrm{~nm}$. The cells ignored laser spots with $\lambda=540 \mathrm{~nm}$ which is consistent with the fact that the water absorption coefficient $\alpha_{540 \mathrm{~nm}}$ at this wavelength is two orders of magnitude lower than $\alpha_{800 \mathrm{~nm}}$ at $\lambda=800 \mathrm{~nm}$ (Pope and Fry, 1997), implying a negligible laser-induced heating. Similarly, it was observed that lamellipodia extension also decreased for laser spots with $\lambda \geq 1200 \mathrm{~nm}$. Since $\alpha_{1200 \mathrm{~nm}}$ is an order of magnitude larger than $\alpha_{1064 \mathrm{~nm}}$ it is possible that irradiating the sample with lasers having $\lambda \geq 1200 \mathrm{~nm}$ simply heats the cell too much, causing massive membrane depolarization and large $\mathrm{Ca}^{2+}$-influx, implying retraction due to very large cytoplasmic $\mathrm{Ca}^{2+}$ concentration (Kou et al., 1993; Albrecht-Buehler, 1991; Henley and Poo, 2004).

\section{Proposed experimental designs}

As temperature effects have previously been neglected in the literature, no systematic experimental studies relating success rates of the optical neuronal guidance to parameters of laser heating exist in the literature. We therefore propose two experimental designs to help providing such data and to test our hypothesis that optical neuronal guidance is mediated by heat-induced TRP channel $\mathrm{Ca}^{2+}$-currents across the neuronal growth cone.

To only test the involvement of the TRP channels, a possibility is to redo neuronal guidance experiments with TRP knockout neurons, or with neurons incubated with a TRP channel blocker (i.e. SKF-96364 (Wang and Poo, 2005)), or with a zero concentration of extracellular $\mathrm{Ca}^{2+}$ (e.g. by substitution with $\mathrm{Mg}^{2+}$ ). This would 

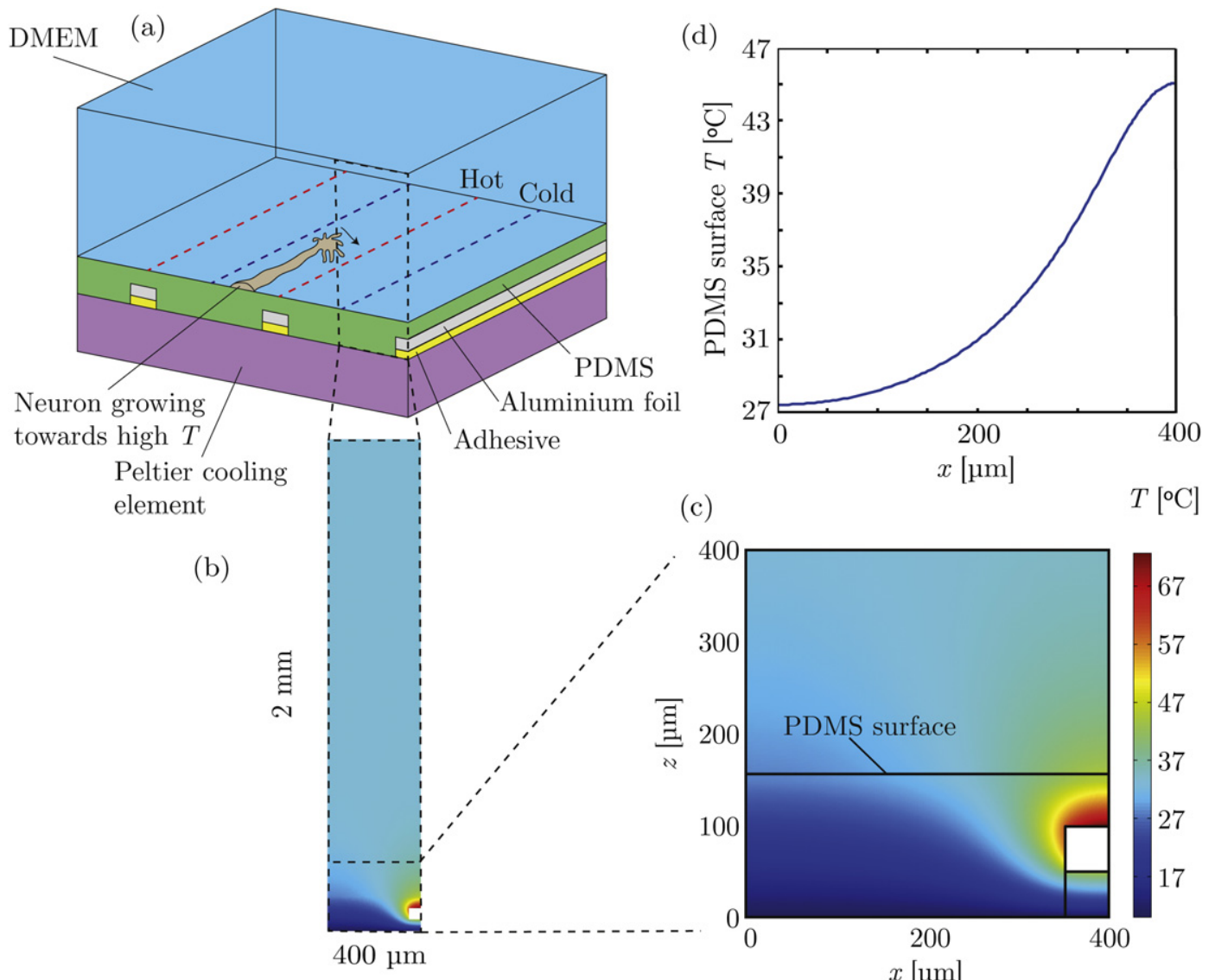

(b)

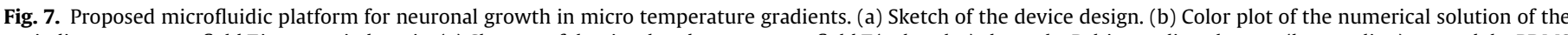

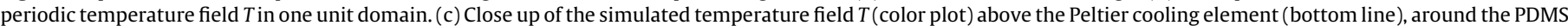

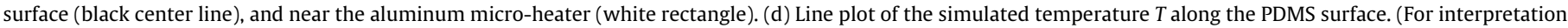
of the references to color in this figure legend, the reader is referred to the web version of the article.)

make it possible to test the involvement of $\mathrm{Ca}^{2+}$ and TRP channels in the sensing mechanism.

To test if it is possible to alter the direction of the neuronal growth using only localized cell heating and no laser light, we propose to incubate growing neurons in temperature gradients on the micrometer scale, similar in magnitude to those, we have found in our laser heating analysis above. In Fig. 7(a) we present a design of a microfluidic platform for this purpose. The device consists of a standard Peltier cooling element kept at a constant temperature $T_{\mathrm{Pel}}$. This can be achieved as in Ref. (Ebbesen et al., 2010) by using a resistive thermal device (RTD) in a feedback loop controlling the current through the Peltier cooling element. On top of the Peltier cooling element, a number of parallel $100 \mu \mathrm{m}$ wide strips of commercially available adhesive aluminum foil are attached. The thickness of both the foil and the adhesive is $100 \mu \mathrm{m}$. The aluminum strips are attached to a current source and serve as Joule micro-heaters when carrying a current $I_{\mathrm{Al}}$. On top of the Peltier cooling element and aluminum micro-heaters, an even layer of PDMS (polydimethylsiloxane) is deposited. This could be achieved using evaporation or spin-coating techniques (possibly in conjunction with flat-pressure), and should be very flat to keep the PDMS surface free of any topological variations to influence the neuronal growth.

The experiment would be carried out by placing the device in DMEM, seeding neurons on the PDMS surface and incubating them at $37^{\circ} \mathrm{C}$, thereby allowing them to start extending growth cones. If the Peltier cooling element and the aluminum micro-heaters are simultaneously turned on, steady-state temperature gradients on the PDMS surface will develop. By allowing the neurons to grow in these gradients, it would be possible to see if the neurons have a tendency to turn the growth direction along the temperature gradients. By constructing a device with many parallel micro-heaters, it is possible to set up many steady-state temperature gradients on the PDMS surface, and carry out numerous "thermal guidance" experiments in parallel, allowing for a large amount of data and good statistics.

In Fig. 7(c) we show a 2D simulation of the temperature field around an aluminum micro-heater. A 2D model is sufficient due to the translational invariance of along the length of the microheaters and the periodicity of the parallel micro-heaters. As above, the calculation was done using COMSOL by solving the steady-state, source-free heat equation,

$-\kappa \nabla^{2} T(\mathbf{r})=0$,

in the PDMS, water, and DMEM domains. The only heat source are the aluminum heaters, but these are just modeled as boundary condition injecting a heat flux due to their Joule heating,

$\mathbf{n} \cdot(\kappa \nabla T)=J_{\mathrm{Al}}$,

where $J_{\mathrm{Al}}$ is the heat flux out of the micro-heaters. We can find $J_{\mathrm{Al}}$ by considering an element of micro-heater of height $h$ and width $w$. Then we have the heat flux,

$J_{\mathrm{Al}}=\frac{I_{\mathrm{Al}}^{2} \rho_{\mathrm{Al}}}{2 h w(h+w)}$,

where $\rho_{\mathrm{Al}}=1.72 \times 10^{-8} \Omega \mathrm{m}$ is the resistivity of aluminum, and where the electric current $I_{\mathrm{Al}}$ through the heater strips is externally 
controllable. In out case, we have $h=50 \mu \mathrm{m}$ and $w=100 \mu \mathrm{m}$. At the top of the DMEM, we will allow heat exchange with air, given by Eq. (6), and due to the feedback regulation of the Peltier cooling element, we will require that the top of the Peltier cooling element will be kept constant at $T_{\mathrm{Pel}}$. Due to the many parallel heaters, we require mirror-symmetry at the vertical boundaries. In Fig. 7(b) and (c) we show a simulation of the steady-state temperature field around an aluminum micro-heater calculated using $T_{\mathrm{Pel}}=10^{\circ} \mathrm{C}, T_{\mathrm{air}}=29^{\circ} \mathrm{C}$, $I_{\mathrm{Al}}=2.8 \mathrm{~A}$, and a total DMEM depth of $1.85 \mathrm{~mm}$. We have used that $\kappa_{\text {DMEM }} \approx \kappa_{\text {wa }}, \kappa_{\text {PDMS }}=0.3 \mathrm{~W} / \mathrm{Km}$ and $\kappa_{\text {adhesive }} \approx \kappa_{\text {epoxy }}=0.2 \mathrm{~W} / \mathrm{Km}$. In Fig. 7(d) we show a line plot of the temperature of the PDMS surface. We find temperature variation between $27^{\circ} \mathrm{C}$ and $45^{\circ} \mathrm{C}$ with temperature gradients comparable to optical guidance experiments over length-scales comparable to the size of neuronal growth cones. An convergence analysis similar to the one mentioned in Section 3.4 was done to ensure the convergence of the numerical solution.

\section{Conclusions}

By numerical simulation using published input parameters, we have shown that in optical neuronal guidance experiments, biologically significant temperature gradients exist on length scales comparable to the size of neuronal growth cones.

We have tied the existence of significant thermal gradients to our proposing of a new hypothesis: Growth cone steering in optical neuronal guidance is mediated by heat-activation of the TRP channels. We have shown that this hypothesis is consistent with previously reported experiments, and that in particular it can explain observed effects of the optical neuronal guidance, which cannot be explained by previous published hypotheses relying only on optical gradient forces (growth cone retraction and dependence of beam shapes).

Finally, we have presented two experiments to test our TRP channel activation hypothesis: one using standard methods from molecular biology involving TRP knockout neurons or a TRP channel blocker in optical guidance experiments, and one physical involving microheaters and no lasers. We have designed a microfluidic platform, which could easily be made using standard microfabrication techniques, for incubating neurons in temperature gradients on micrometer lengthscales, which would be suitable for carrying out the latter test.

If the optical neuronal guidance is indeed a result of TRP channel activation, it is a rudimentary form of cellular thermo-sensing, which may find novel applications in bioengineering, cellular biology and regenerative medicine.

\section{References}

Albrecht-Buehler G. Surface extensions of 3T3 cells towards distant infrared light sources. J Cell Biol 1991:114:493-502.

Andersen SS, Jackson AD, Heimburg T. Towards a thermodynamic theory of nerve pulse propagation. Prog Neurobiol 2009;88:104-13.

Atkinson SJ, Hosford MA, Molitoris BA. Mechanism of actin polymerization in cellular ATP depletion. J Biol Chem 2004;279:5194-9.

Blicher A, Wodzinska K, Fidorra M, Winterhalter M, Heimburg T. The temperature dependence of lipid membrane permeability, its quantized nature, and the influence of anesthetics. Biophys J 2009;96:4581-91.

Braun D, Libchaber A. Trapping of DNA thermophoretic depletion and convection. Phys Rev Lett 2002;89, 188103-1-4.

Bruus H. Theoretical microfluidics. 2nd ed. Oxford: Oxford University Press; 2008

Carnegie D, Cizmar T, Baumgartl J, Gunn-Moore F, Dholakia K. Automated laser guidance of neuronal growth cones using a spatial light modulator. J Biophoton 2009;2:682-92.

Carnegie D, Stevenson D, Mazilu M, Gunn-Moore F, Dholakia K. Guided neuronal growth using optical line traps. Opt Express 2008;16:10507-17.

Caterina MJ, Schumacher Ma, Tominaga M, Rosen Ta, Levine JD, Julius D. The capsaicin receptor: a heat-activated ion channel in the pain pathway. Nature 1997;389:816-24.

Caterina MJ. Transient receptor potential ion channels as participants in thermosensation and thermoregulation. Am J Physiol-Reg Integr Comp 2007;292:R64-76.
Chen S, Cheng $\mathrm{H}$. Functions of axon guidance molecules in synapse formation. Curr Opin Neurobiol 2009;19:471-8.

COMSOL Comsol 4.2 multiphysics finite element analysis software, 2011 http://www.comsol.com/.

Ebbesen CL, Adams JD, Barnkob R, Soh HT, Bruus H. Temperature-controlled highthroughput $(1 \mathrm{~L} / \mathrm{h})$ acoustophoretic particle separation in microchannels. In: Verporte S, Andersson H, Emneus J, Pamme N, editors. Proc 14th MicroTAS, 3-7 October 2010, Groningen, The Netherlands; 2010. p. 728-30, CBMS.

Ebert S, Travis K, Lincoln B, Guck J. Flourescence ratio thermometry in a microfluidic dual-beam laser trap. Opt Express 2007;15:15493-9.

Ehrlicher A, Betz T, Stuhrmann B, Koch D, Milner V, Raizen M, et al. Guiding neuronal growth with light. Proc Natl Acad Sci USA 2002;99:16024-8.

Franze K, Guck J. The biophysics of neuronal growth. Rep Prog Phys 2010;73:094601.

Fukui Y, Hoshino K, Inouye M, Kameyama Y. Effects of hyperthermia induced by microwave irradiation on brain-development in mice. J Radiat Res 1992;33:1-10.

Graves C, McAllister R, Rosoff W, Urbach J. Optical neuronal guidance in threedimensional matrices. J Neurosci Methods 2009;179:278-83.

Henley J, Poo M. Guiding neuronal growth cones using Ca2+signals. Trends Cell Biol 2004; $14: 320-30$.

Henley J, Huang K, Wang D, Poo M. Calcium mediates bidirectional growth cone turning induced by myelin-associated glycoprotein. Neuron 2004;44:909-16.

Higuchi A, Watanabe T, Matsubara Y, Matsuoka Y, Hayashi S. Regulation of neurite outgrowth by intermittent irradiation of visible light. J Phys Chem B 2005;109:11033-6.

Higuchi A, Watanabe T, Noguchi Y, Chang Y, Chen W, Matsuoka Y. Visible light regulates neurite outgrowth of nerve cells. Cytotechnology 2007;54:181-8.

Hong K, Nishiyama M, Henley J, Tessier-Lavigne M, Poo M. Calcium signalling in the guidance of nerve growth by netrin-1. Nature 2000;403:93-8.

Huang H, Delikanli S, Zeng H, Ferkey DM, Pralle A. Remote control of ion channels and neurons through magnetic-field heating of nanoparticles. Nat Nanotechno 2010;5:602-6.

Irie T, Hara M, Yasukura T, Minamino M, Omori K, Matsuda H, et al. Chloride concentration in cultured hippocampal neurons increases during long-term exposure to ammonia through enhanced expression of an anion exchanger. Brain Res 1998;806:246-56.

Ishida A, Cheng M. Cold inhibits neurite outgrowth from single retinal ganglion-cells isolated from adult goldfish. Exp Eye Res 1991;52:175-91.

Kater S, Guthrie P. Neuronal growth cone as an integrator of complex environmenta information. Cold Spring Harbor Symp Quant Biol 1990;55:359-70.

Koch D, Betz T, Ehrlicher A, Gogler M, Stuhrmann B, Kas J. Optical control of neuronal growth. Proc SPIE 2004;5514:428-36.

Kou L, Labrie D, Chylek P. Refractive indices of water and ice in the 0.65 to $2.5 \mu \mathrm{m}$ spectral range. Appl Opt 1993;32:3531-40.

Li Y, Jia YC, Cui K, Li N, Zheng ZY, Wang Yz, et al. Essential role of TRPC channels in the guidance of nerve growth cones by brain-derived neurotrophic factor. Nature 2005;434:894-8

Lide DR, editor. Handbook of chemistry and physics. 84th ed. Boca-Raton: CRC Press 2003.

Liu Y, Cheng D, Sonek G, Berns M, Chapman C, Tromberg B. Evidence for localized cell heating induced by infrared optical tweezers. Biophys J 1995;68:2137-44.

Mathew M, Amat-Roldan I, Andres R, Santos SICO, Artigas D, Soriano E, et al Signalling effect of NIR pulsed lasers on axonal growth. J Neurosci Methods 2010;186:196-201.

Mochizuki-Oda N, Kusuno T, Yamashita H, Takamori Y, Yamada H, Yamada H. Warm temperature-activated $\mathrm{Ca} 2+$ response in cultured fibroblasts, neuronal cells and keratinocytes. Neurosci Res 2007;58:S195.

Mohanty S, Sharma M, Panicker M, Gupta P. Controlled induction, enhancement and guidance of neuronal growth cones by use of line optical tweezers. Opt Let 2005:30:2596-8.

Niranjan P, Forbes J, Greer S, Dudowicz J, Freed K, Douglas J. Thermodynamic regulation of actin polymerization. J Chem Phys 2001;114:10573-6.

Nishiyama M, Hoshino A, Tsai L, Henley J, Goshima Y, Tessier-Lavigne M, et al. Cyclic AMP/GMP-dependent modulation of $\mathrm{Ca} 2+$ channels sets the polarity of nerve growth-cone turning. Nature 2003;423:990-5.

Peterman EJG, Gittes F, Schmidt CF. Laser-induced heating in optical traps. Biophys J 2003:84:1308-16.

Pline M, Diez Ja, Dusenbery DB. Extremely sensitive thermotaxis of the nematode meloidogyne incognita. J Nematol 1988;20:605-8.

Pope RM, Fry ES. Absorption spectrum $(380-700 \mathrm{~nm})$ of pure water. II. Integrating cavity measurements. Appl Opt 1997;36:8710-23.

Sappington RM, Sidorova T, Long DJ, Calkins DJ. TRPV1: contribution to retina ganglion cell apoptosis and increased intracellular Ca2+with exposure to hydrostatic pressure. Invest Ophthalmol Vis Sci 2009;50:717-28.

Schönle A, Hell SW. Heating by adsorption in the focus of an objective lens. Opt Let 1998;23:325-7

Stevenson DJ, Gunn-Moore F, Dholakia K. Light forces the pace: optical manipulation for biophotonics. J Biomed Opt 2010;15, 041503-1-21.

Stevenson D, Lake T, Agate B, Garces-Chavez V, Dholakia K, Gunn-Moore F. Optically guided neuronal growth at near infrared wavelengths. Opt Express 2006;14:9786-93.

Szallasi A, Cortright DN, Blum CA, Eid SR. The vanilloid receptor TRPV1: 10 years from channel cloning to antagonist proof-of-concept. Nat Rev Drug Discov 2007;6:357-72

Talavera K, Nilius B, Voets T. Neuronal TRP channels: thermometers, pathfinders and life-savers. Trends Neurosci 2008;31:287-95. 
Wang G, Poo M. Requirement of TRPC channels in netrin-1-induced chemotropic turning of nerve growth cones. Nature 2005;434:898-904.

Wodzinska K, Blicher A, Heimburg T. The thermodynamics of lipid ion channel formation in the absence and presence of anesthetics. BLM experiments and simulations. Soft Matter 2009;5:3319-29.

Wunderlich B, Leirer C, Idzko A, Keyser U, Wixforth A, Myles V, et al. PhaseState dependent current fluctuations in pure lipid membranes. Biophys J 2009;96:4592-7.
Zeck G, Fromherz P. Noninvasive neuroelectronic interfacing with synaptically connected snail neurons immobilized on a semiconductor chip. Proc Natl Acad Sci USA 2001;98:10457-62.

Zimmerle CT, Frieden C. Effect of temperature on the mechanism of actin polymerization. Biochemistry 1986;25:6432-8. 